REMARKS. (1) It is easy to construct examples of rings which satisfy condition $\left(b_{l}\right)$ but not condition $\left(a_{l}\right)$.

(2) Any ring which satisfies condition $\left(a_{l}\right)$ and contains a minimal left ideal must have $(L)^{0} \neq R$ for every minimal left ideal.

The author wishes to thank Professors B. Vinograde and T. J. Head for their help in organizing this note.

\title{
BIBLIOGRAPHY
}

1. J. Dieudonne, Remarks on quasi-Frobenius rings, Illinois J. Math. 2 (1958), 346-354.

2. M. Ikeda, A characterization of quasi-Frobenius rings, Osaka Math. J. 4 (1952), 203-209.

IOWA STATE University

\section{ON THE SUBGROUPS OF THE PICARD GROUP'}

HERMANN V. WALDINGER

1. Introduction. The Picard group $\Gamma$ is important in the Theory of Automorphic Functions [3]. It consists of all linear transformations

$$
w=\frac{a z+b}{c z+d}, \quad a d-b c= \pm 1
$$

with coefficients Gaussian integers. $\Gamma$ is known [3] to have four generators

$$
s, t, u, v
$$

together with the eight defining relations

(1.3) $s^{2}=u^{2}=v^{2}=\left(u s^{-1}\right)^{2}=\left(v t^{-1}\right)^{2}=\left(s t^{-1}\right)^{2}=\left(u t^{-1}\right)^{3}=\left(v u^{-1}\right)^{3}=1$.

The generators (1.2) are the transformations $w=-z, w=z-1$, $w=-1 / z$, and $w=-z+i$ respectively.

In this paper, we seek to examine the structure of the Picard group by studying its subgroups. The modular group is a well-known subgroup. It consists of all transformations (1.1) with coefficients

Received by the editors November 19, 1964.

1 This paper was completed under NSF grant GP-3204. 
rational integers. The subgroups of the modular group have been investigated extensively [6], [7], [8]. In particular they have been studied as abstract groups given by generators and defining relations [8]. We do not attempt here to make general statements about all subgroups of the Picard group. Instead we shall consider only special subgroups.

In particular we shall construct the subgroup $H$ of index 48 which has a remarkably simple structure. It is the free product of two isomorphic groups $J_{L}$ and $J_{R}$ with the amalgamated free subgroup $J$. Moreover $J_{L}$ is the free product of two isomorphic free abelian groups of rank two.

We conclude this paper with a short discussion of Fuchsian subgroups.

2. The subgroup $H$. To find $H$, we compute the following chain of subgroups by an algorithm due to O. Schreier [5]:

a. $\Gamma_{1}=$ commutator subgroup of $\Gamma$.

b. $\Gamma_{2}=$ commutator subgroup of $\Gamma_{1}$.

$\Gamma_{2}$ has six generators $x_{1}, x_{2}, \cdots, x_{6}$.

c. $F=$ subgroup of $\Gamma_{2}$ consisting of all words of even exponentsum in $x_{1}, x_{2}, \cdots, x_{6}$.

$F$ has five generators $y_{1}, y_{2}, \cdots, y_{5}$.

d. $H=$ subgroup of $F$ consisting of all words of even exponentsum in $y_{1}, y_{2}, \cdots, y_{5}$.

Before discussing our chain let us first review Schreier's method [5] of finding the representation of a subgroup by generators and defining relations.

Let $G$ be a group given by the generators $g_{i}$ and the defining relations $f_{j}\left(g_{i}\right)=1$, where the $f_{j}\left(g_{i}\right)$ are products of the powers of the $g_{i}$. Let $U$ be a subgroup of $G$. Let $\bar{G}$ be the free group generated by the $g_{i}$. Let $\widetilde{U}$ be that subgroup of $\widetilde{G}$ consisting of all words in the $g_{i}$ which as elements of $G, \in U$. Evidently

$$
[G: U]=[\tilde{G}: \tilde{U}] \text {. }
$$

Furthermore, if $N$ is the normal closure in $\widetilde{G}$ of the subgroup generated by the $f_{j}$, then

$$
G \cong \tilde{G} / N, \quad U \cong \tilde{U} / N
$$

Our method starts out by assigning to every $g \in \tilde{G}$ a representative, $\bar{g}$, of its coset $\tilde{U} \bar{g}$ subject to the following conditions:

The representative of $\tilde{U}$ is 1 .

If $\prod_{k=1}^{m} g_{k}^{\epsilon}\left(m>1, \epsilon_{k}= \pm 1\right)$ is a representative, then $\prod_{k=1}^{m-1} g_{k}^{\epsilon_{k}}$ is also a representative. 
Having found the $\bar{g}$, we finally compute the generators and defining relations of $U$ according to the rules:

The set of generators consists of all $\bar{g} g_{i}\left(\bar{h}_{i}\right)^{-1}$, where $h_{i}=g g_{i}$.

The set of defining relations is given by setting all expressions $\bar{g} f_{j}\left(g_{i}\right)(\bar{g})^{-1}=1$.

We are now ready to apply the Schreier procedure.

It is not difficult to see that $\left[\tilde{\Gamma}: \tilde{\Gamma}_{1}\right]=4$ and that the cosets have the representatives $1, s, u, s u$. A straightforward, but lengthy computation yields then

LEMMA 1. $\left[\Gamma: \Gamma_{1}\right]=4$. $\Gamma_{1}$ is given by the generators

$$
u_{1}=t u^{-1}, \quad u_{2}=s t u^{-1} s^{-1}, \quad u_{3}=v u^{-1}, \quad u_{4}=s v u^{-1} s^{-1}
$$

together with the defining relations

$$
\begin{aligned}
u_{1}^{3} & =u_{2}^{3}=u_{3}^{3}=u_{4}^{3}=1, \\
\left(u_{1} u_{3}^{-1}\right)^{2} & =\left(u_{1} u_{4}^{-1}\right)^{2}=\left(u_{2} u_{3}^{-1}\right)^{2}=\left(u_{2} u_{4}^{-1}\right)^{2}=1 .
\end{aligned}
$$

Next let us compute $\Gamma_{2}$. It is evident that $\left[\tilde{\Gamma}_{1}: \tilde{\Gamma}_{2}\right]=3$ and that $1, u_{1}, u_{1}^{2}$ are coset representatives. By our procedure we obtain at once the 10 generators

$$
\begin{aligned}
& u_{1}^{3}, u_{2} u_{1}^{-1}, u_{1} u_{2} u_{1}^{-2}, u_{1}^{2} u_{2}, u_{3} u_{1}^{-1}, \\
& u_{1} u_{3} u_{1}^{-2}, u_{1}^{2} u_{3}, u_{4} u_{1}^{-1}, u_{1} u_{4} u_{1}^{-2}, u_{1}^{2} u_{4}
\end{aligned}
$$

together with 16 relations. But the generators $u_{1}^{3}, u_{1}^{2} u_{2}, u_{1}^{2} u_{3}$ and $u_{1}^{2} u_{4}$ are eliminated immediately by four relations. Let us replace the remaining 6 generators by

$$
\begin{aligned}
& a=u_{2} u_{3}^{-1}, \quad b=u_{1} u_{2} u_{3}^{-1} u_{1}^{-1}, \quad c=u_{3} u_{1}^{-1}, \\
& d=u_{1} u_{3} u_{1}^{-2}, \quad e=u_{4} u_{1}^{-1}, \quad f=u_{1} u_{4} u_{1}^{-2} .
\end{aligned}
$$

We then obtain the 12 relations

$$
\begin{aligned}
a^{2} & =b^{2}=c^{2}=d^{2}=e^{2}=f^{2}=1 . \\
(a c e)^{2} & =(b d f)^{2}=(c d)^{2}=(e f)^{2}=(b c a c)^{2} \\
& =(b c a \text { e } f d)^{2}=1 .
\end{aligned}
$$

But to proceed to the subgroup $H$ we must also replace the generators (2.6). We arrive at

LEMMA 2. $\left[\Gamma: \Gamma_{2}\right]=12 . \Gamma_{2}$ is given by the 6 generators

(2.8) $x_{1}=c a e, x_{2}=e, x_{3}=c, x_{4}=f d b, x_{5}=f, x_{6}=d$

\section{together with the relations}




$$
\begin{aligned}
x_{1}^{2} & =x_{2}^{2}=x_{3}^{2}=x_{4}^{2}=x_{5}^{2}=x_{6}^{2}=\left(x_{1} x_{4}\right)^{2}=\left(x_{2} x_{5}\right)^{2}=\left(x_{3} x_{6}\right)^{2} \\
& =\left(x_{1} x_{2} x_{3}\right)^{2}=\left(x_{4} x_{5} x_{6}\right)^{2}=\left(x_{1} x_{2} x_{3} x_{4} x_{5} x_{6}\right)^{2}=1 .
\end{aligned}
$$

We proceed now to the computation of $F$. Evidently $\left[\tilde{\Gamma}_{2}: \tilde{F}\right]=2$ and 1 and $x_{2}$ are coset representatives. The Schreier procedure gives at once the 11 generators

$$
x_{1} x_{2}^{-1}, x_{3} x_{2}^{-1}, x_{4} x_{2}^{-1}, x_{5} x_{2}^{-1}, x_{6} x_{2}^{-1}, x_{2} x_{1}, x_{2}^{2}, x_{2} x_{3}, x_{2} x_{4}, x_{2} x_{5}, x_{2} x_{6}
$$

together with 12 relations. Eliminating the six generators $x_{1} x_{2}^{-1}$, $x_{3} x_{2}^{-1}, x_{4} x_{2}^{-1}, x_{5} x_{2}^{-1}, x_{6} x_{2}^{-1}, x_{2}^{2}$ by six of the relations and replacing $x_{2} x_{1}$, $x_{2} x_{3}$ by $x_{5} x_{1}, x_{5} x_{3}$ we obtain

LEMмA 3. $[\Gamma: F]=24 . F$ is given by the 5 generators

$$
y_{1}=x_{5} x_{1}, y_{2}=x_{5} x_{3}, y_{3}=x_{2} x_{4}, y_{4}=x_{2} x_{5}, y_{5}=x_{2} x_{6}
$$

together with the six relations

$$
\begin{aligned}
y_{3}^{2} & =\left(y_{4}^{-1} y_{8} y_{1}\right)^{2}=\left(y_{5}^{-1} y_{3} y_{2}\right)^{2}=\left(y_{3} y_{1}\right)\left(y_{3} y_{2}\right)\left(y_{3} y_{1}\right)^{-1}\left(y_{3} y_{2}\right)^{-1} \\
& =\left(y_{3} y_{4}\right)\left(y_{3} y_{6}\right)\left(y_{3} y_{4}\right)^{-1}\left(y_{3} y_{5}\right)^{-1} \\
& =\left(\left(y_{3} y_{1}\right)\left(y_{3} y_{2}\right)\left(y_{3} y_{6}\right)^{-1}\left(y_{3} y_{4}\right)^{-1} y_{3}\right)^{2}=1 .
\end{aligned}
$$

We are at last ready to compute $H$. We note that $[\tilde{F}: \tilde{H}]=2$ and that 1 and $y_{3}$ are coset representatives. By our procedure we obtain then the generators $y_{1} y_{3}^{-1}, y_{3} y_{1}, y_{2} y_{3}^{-1}, y_{3} y_{2}, y_{4} y_{3}^{-1}, y_{3} y_{4}, y_{6} y_{3}^{-1}, y_{8} y_{6}$ together with seven relations. To obtain our result we introduce the new generators

$$
\begin{aligned}
& a_{L}=y_{4} y_{3}^{-1}, \quad b_{L}=y_{5} y_{3}^{-1}, \quad \alpha_{L}=y_{3} y_{1}^{-1}, \quad \beta_{L}=y_{8} y_{2}^{-1}, \\
& a_{R}=y_{8} y_{1}, \quad b_{R}=y_{8} y_{2}, \quad \alpha_{R}=y_{4}^{-1} y_{3}^{-1}, \quad \beta_{R}=y_{5}^{-1} y_{3}^{-1}
\end{aligned}
$$

for which we have the defining relations

$$
\begin{aligned}
a_{L} b_{L} a_{L}^{-1} b_{L}^{-1} & =a_{R} b_{R} a_{R}^{-1} b_{R}^{-1}=\alpha_{L} \beta_{L} \alpha_{L}^{-1} \beta_{L}^{-1}=\alpha_{R} \beta_{R} \alpha_{R}^{-1} \beta_{R}^{-1}=1, \\
a_{L} \alpha_{L} & =a_{R} \alpha_{R}, b_{L} \beta_{L}=b_{R} \beta_{R}, a_{L} b_{L} \alpha_{L} \beta_{L}=a_{R} b_{R} \alpha_{R} \beta_{R} .
\end{aligned}
$$

We have established the following theorem.

TheOREM 1. $[\Gamma: H]=48 . H$ is the free product of isomorphic groups $J_{L}$ and $J_{R}$ with the amalgamated subgroup $J_{.} J_{L}$ is the free product of two free abelian groups generated by $a_{L}, b_{L}$ and $\alpha_{L}, \beta_{L}$ respectively. The isomorphism between $J_{L}$ and $J_{R}$ is given by the correspondence

$$
a_{L} \leftrightarrow a_{R}, \quad b_{L} \leftrightarrow b_{R}, \quad \alpha_{L} \leftrightarrow \alpha_{R}, \quad \beta_{L} \leftrightarrow \beta_{R} .
$$


The amalgamated subgroup $J$ is generated by

$$
a_{L} \alpha_{L}=a_{R} \alpha_{R}, b_{L} \beta_{L}=b_{R} \beta_{R}, a_{L} b_{L} \alpha_{L} \beta_{L}=a_{R} b_{R} \alpha_{R} \beta_{R} .
$$

It is free of rank three.

In concluding the discussion of $H$ we note a remarkable property of the amalgamated subgroup $J$. It is not difficult to show that $J_{N}$, the normal closure of $J$ in $\Gamma$ has infinite order and infinite index. This fact is of interest in connection with a recent paper [1]. It is shown there that such subgroups do not exist for a large class of linear groups on more than two variables.

3. The Fuchsian subgroups. We study here the normal closures of Fuchsian subgroups. In particular we seek to determine their indices in $\Gamma$.

We start with preliminary definitions and the statement of a known result.

Let $\mathfrak{C}$ be a circle in the complex plane given by

$$
\begin{aligned}
& a z \bar{z}+\left(b_{1}-i b_{2}\right) z+\left(b_{1}+i b_{2}\right) \bar{z}+c=0, \\
& a, b_{1}, b_{2}, c \text { real, } \\
& b_{1}^{2}+b_{2}^{2}-a c>0 .
\end{aligned}
$$

We shall say that a Fuchsian subgroup $P$ of $\Gamma$ belongs to the circle $\mathcal{C}$, if the transformations of $P$ map both the interior and the boundaries of $\mathfrak{C}$ onto themselves. For a given $\mathfrak{C}$ we shall denote the corresponding Fuchsian subgroup by $P(\mathbb{C})$ and its normal closure by $P_{N}(\mathfrak{C})$. The following is known [3]:

Let $\{a\}$ be the class of circles $\mathcal{C}$ for which the coefficients $a, b_{1}, b_{2}, c$ of (3.1) are rational integers. Every Fuchsian subgroup $P$ belongs then to a circle of $\{R\}$ and conversely to every such circle there belongs a Fuchsian subgroup.

Normal subgroups of finite index in linear groups were investigated a short time ago [1]. In this connection it would be interesting to determine whether $\left[\Gamma: P_{N}(\mathcal{C})\right]$ is finite for all circles $\mathcal{C} \in\{\Re\}$, a task which appears very difficult. In this paper we establish a result only for circles in six subclasses $\left\{R_{i}\right\} \subset\{Q\}$. Each subclass $\left\{R_{i}\right\}$ consists of all circles which are conjugate to representative circles $\mathbb{C}_{i} \in\left\{Q_{i}\right\}$. Two circles are conjugate if they are mapped onto each other by transformations of the Picard group. For $\mathcal{e} \in\left\{\Omega_{i}\right\}$ it is then evident that $P_{N}(\mathbb{e})$ is identical with some $P_{N}\left(\mathfrak{C}_{i}\right)$.

Let us now define the representative circles: For $1 \leqq i \leqq 4$, the $\mathfrak{C}_{i}$ are all circles of $\{R\}$ with center at $q_{i}$ where $q_{1}=0, q_{2}=1 / 2, q_{3}=i / 2$, $q_{4}=(1+i) / 2$. The $e_{5}$ are all lines $\in\{a\}$ parallel to the real axis, the $\mathfrak{C}_{6}$ all such lines parallel to the imaginary axis. 
We are finally ready to investigate $\left[\Gamma: P_{N}(\mathcal{C})\right]$ for circles in the subclasses.

Let $g_{1}, g_{2}, \ldots$ be the elements $\in P(\mathfrak{C})$. Let $\Gamma^{*}$ be the group obtained from $\Gamma$ by superimposing the relations

$$
g_{1}=g_{2}=\cdots=1
$$

upon the defining relations (1.3). Clearly $\tilde{\Gamma} \cong \Gamma / P_{N}(\mathfrak{C})$.

Thus if $\Gamma^{f}$ has $\mu$ elements, then $\left(\Gamma: P_{N}(\mathcal{C})\right)=\mu$.

In the procedure below we shall pick an element $g \in P(\mathcal{C})$ and add $g=1$ to the relations (1.3) to give the group $\Gamma^{\sharp}$. It is evident that $\Gamma^{\sharp}$ is a homomorphic image of $\Gamma \boldsymbol{H}$. We conclude that $\mu$ divides $\nu$, where $\nu$ is the order of $\Gamma \boldsymbol{H}$.

Since every $\mathcal{C} \in\left\{\mathscr{R}_{i}\right\}$ is conjugate to a $\mathfrak{C}_{i}$ it is evidently sufficient to consider the $\mathfrak{C}_{i}$ only.

Now $\mathfrak{C}_{i}(1 \leqq i \leqq 4)$ has center at $q_{i}$ and then $P\left(\mathfrak{C}_{i}\right)$ contains the transformation $d_{i}$, where $d_{1}=s, d_{2}=s t, d_{3}=v, d_{4}=t^{-1} v$. Since $\mathfrak{C}_{5}$ is parallel to the real, and $\mathfrak{e}_{6}$ parallel to the imaginary axis, $P\left(\mathfrak{C}_{5}\right)$ contains $d_{5}=t$ and $P\left(\mathfrak{e}_{6}\right)$ contains $d_{6}=s v$. Setting $d_{i}=1$ we find that $\Gamma$ has the 24 elements $1, u, v, t, u v, u t, v u, v t, t u, u v u, u v t, u t u, v u t$, vtu, tuv, uvut, uvtu, utuv, vutu, vtuv, uvutu, uvtuv, vutuv and uvutuv when $i=1$, the 2 elements 1 and $s$ when $i=2,3,5$ and 6 , finally the 12 elements $1, s, u, t, u t, t u, u t u, s u, s t, s u t, s t u$, and $s u t u$ when $i=4$.

We have established the following theorem:

THEOREM 2. [ $\left.\Gamma: P_{N}(\mathcal{C})\right]$ divides $\nu_{i}$ when $\mathcal{C} \in\left\{Q_{i}\right\}$, where $\nu_{1}=24$, $\nu_{2}=\nu_{3}=\nu_{5}=\nu_{6}=2$ and $\nu_{4}=12$.

\section{REFERENCES}

1. H. Bass, M. Lazard, et J.-P. Serre, Sous-groupes d'indice fini dans $\operatorname{SL}(n, Z)$, Bull. Amer. Math. Soc. 70 (1964), 385-392.

2. L. R. Ford, Automorphic functions, Chelsea, New York, 1929.

3. R. Fricke and F. Klein, Vorlesungen über die Theorie der automorphen Funktionen, Teubner, Leipzig, 1897.

4. M. Hall, The theory of groups, Macmillan, New York, 1959.

5. W. Hurewicz, $Z$ u einer Arbeit Von O. Schreier, Abh. Math. Sem. Univ. Hansischen 8 (1930), 307-314.

6. F. Klein and R. Fricke, Vorlesungen über die Theorie der Modulfunktionen, Teubner, Leipzig, 1890.

7. J. Lehner, Discontinuous groups and automorphic functions, Math Surveys, No. 8, Amer. Math. Soc., Providence, R. I., 1964.

8. W. Magnus, Untersuchungen über einige unendliche diskontinuirliche Gruppen, Math. Ann. 105 (1931), 52-74.

\section{BrookL yn Polytechnic Institute}

